\title{
Singular Integral Formulations for Electrodynamic Analysis of Metamaterial-Inspired Antenna Array
}

\author{
Mohammad Alibakhshikenari, Member IEEE, Bal S. Virdee, Senior Member IEEE, Sonia Aïssa, Fellow IEEE, \\ Chan H. See, Senior Member IEEE, Ayman A. Althuwayb, Raed Abd-Alhameed, Senior Member IEEE, Isabelle \\ Huynen, Senior Member IEEE, Francisco Falcone, Senior Member IEEE, and Ernesto Limiti, Senior Member
} IEEE

\begin{abstract}
In this paper, a set of singular integral formulations are derived to calculate the surface impedance matrix on the antenna array elements. The proposed mathematical model enables electrodynamic analysis of antenna arrays designed using metamaterial-inspired structures. The formulations allow the determination of the array's impedance, spatial and polarization characteristics at significantly low computational cost compared to conventional electromagnetic solvers based on method-of-moments (MoM) numerical technique. The accuracy of the surface impedance results obtained from the theoretical formulations are verified using the full wave EM software. It is shown that there is excellent agreement between the proposed formulations and EM software. The accuracy of the theoretical model presented is valid for single layer structures.
\end{abstract}

Keywords - Singular integral formulation, metamaterial, antenna array, electrodynamic analysis, EM simulation.

\section{INTRODUCTION}

The use of metamaterial-inspired structures in the design of antennas and antenna arrays is becoming common now [1]. It is evident in literature that the utilization of metamaterial (MTM) can simultaneously improve the antenna's radiation characteristics and reduce its physical footprint [2-5]. Arrays

This work is partially supported by RTI2018-095499-B-C31, Funded by Ministerio de Ciencia, Innovación y Universidades, Gobierno de España (MCIU/AEI/FEDER,UE), and innovation programme under grant agreement H2020-MSCA-ITN-2016 SECRET-722424 and the financial support from the UK Engineering and Physical Sciences Research Council (EPSRC) under grant EP/E022936/1.

M. Alibakhshikenari and E. Limiti are with the Electronic Engineering Department, University of Rome "Tor Vergata", Italy (e-mail: alibakhshikenari@ing.uniroma2.it and limiti@ing.uniroma2.it).

B. S. Virdee, is with the London Metropolitan University, Center for Communications Technology, U.K. (e-mail: b.virdee@londonmet.ac.uk).

S. Aïssa is with the Institut National de la Recherche Scientifique (INRS), University of Quebec, Montreal, Canada (e-mail: aissa@emt.inrs.ca).

C. H. See is with the School of Engineering and the Built Environment, Edinburgh Napier University, Edinburgh, U.K. (e-mail: c.see@napier.ac.uk).

A. A. Althuwayb is with the Electrical Engineering Department, Jouf University, Sakaka, Aljouf, Kingdom of Saudi Arabia (e-mail: aaalthuwayb@ju.edu.sa).

R. Abd-Alhameed is with the Faculty of Engineering and Informatics, University of Bradford, Bradford, UK (e-mail: r.a.a.abd@bradford.ac.uk).

I. Huynen is with the Institute of Information and Communication Technologies, Electronics and Applied Mathematics, Université Catholique de Louvain, Belgium (e-mail: isabelle.huynen@uclouvain.be).

F. Falcone is with the Electric, Electronic and Communication Engineering Department, Public University of Navarre, and Institute of Smart Cities, Public University of Navarre, 31006 Pamplona, Spain (e-mail: francisco.falcone@unavarra.es). are composed of an arrangement of multiple antennas however the proximity of antennas can degrade the performance of arrays due to interactions of surface waves and near-field radiation. It has been shown in [6-7] that antenna arrays that are based on MTM structures exhibit higher isolation between the radiating elements and therefore improved electromagnetic compatibility. The consequence of this is improvement in the array's overall radiation performance. Such arrays have been shown to increase the throughput in MIMO systems [8-10].

Electrodynamic analysis of antennas and antenna arrays is typically carried out using commercial electromagnetic solvers such as CST Studio Suite ${ }^{\circledR}$ that employ finite integration technique (FIT), which is a discretization scheme for Maxwell's formulations in their integral form [11]. The use of this approach is associated with challenges associated with [12], namely, (i) inaccuracy of the constructed model, (ii) choice for optimum sampling rate used, and (iii) intensive demand on computational sources. The alternative method involves the use of method-of-moments (MoM) approach that involves solving Fredholm integral equations of the first kind to enable the determination of the impedance, spatial and polarization characteristics of the array with a high degree of accuracy using less computational effort.

Proposed in this paper is a set of singular integral formulations of electrodynamic analysis to calculate the matrix of surface impedance over an antenna array based on metamaterial-inspired structures. The proposed technique is shown to be highly accurate and requires significantly less computational effort than a commercial electromagnetic solver based on FIT or MoM.

\section{PRoposed MTM ANTENNA ARRAYS}

The proposed antenna array, shown in Fig.1, consists of a regular arrangement of multiple MTM-inspired radiating elements consisting of circular patches. Etched on each radiating patch is a narrow rectangular slot, and the patch is connected to the substrate's ground-plan with a metallic via. The periodic array of subwavelength elements act like resonators or scatters that exhibits metamaterial left-handed properties (negative refractive index) when interacting with EM-fields [13]. This antenna structure was chosen as it operates over a wider bandwidth as will be evident in the results presented later. The array is constructed on a dielectric substrate with thickness $h$ and having electrical parameters $\varepsilon_{2}$, $\mu_{2}$, and $\tan \delta$. The empty space above the substrate is defined by $\varepsilon_{1}$ and $\mu_{1}$. Dielectric and permittivity rations $\varepsilon_{2} / \varepsilon_{1} \& \mu_{2} / \mu_{1}$ 
represent the relative electric permittivities and magnetic permeabilities of two media.

In the analysis it is assumed that the single layer dielectric substrate, shown in Fig.1, on which the antenna array is constructed consists of two columns of regularly arranged circular patches of finite thickness and perfect conductivity. The substrate's length is represented by symbol $l$, the diameter of the circular patches is $D$, inter-space gap between successive patches is $s$, and the gap between the two columns of antennas is represented by $g$.

When the antenna array is stimulated by exposing it to RF energy the resulting currents are distributed over the array such that the resulting electromagnetic (EM) field from the array satisfies the boundary conditions of Maxwell's formulations.

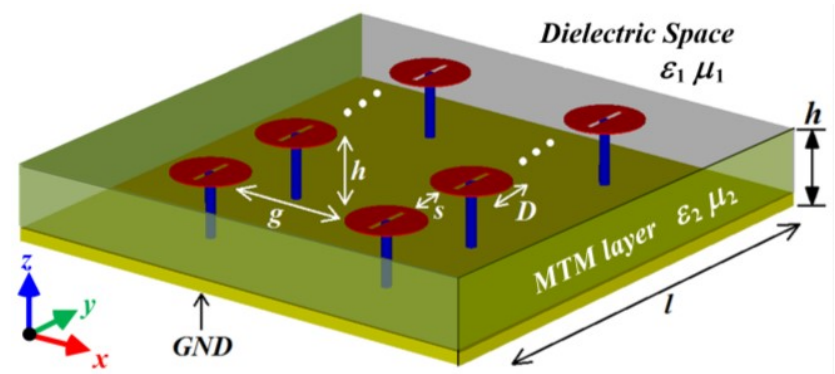

Fig.1. Antenna array realized with metamaterial-inspired circular patch radiators.

\section{IMPEDANCE MATRIX OF THE SURFACE}

To determine surface impedance matrix elements in the antenna array the approach used in [12] first establishes the surface admittance matrix elements $[Y]$ and then converts the matrix to its corresponding $[Z]$ matrix, which takes a longer processing time. Unlike in [12] the proposed methodology used here avoids the complexity of this conversion step and therefore the additional computation processing. This is achieved by directly calculating the surface impedances matrix elements, the consequence of which is reduction in the computational time. Because the computational time in [12] was not reported it was necessary to benchmark the proposed technique against a commercial 3D full wave electromagnetic solver based on MoM. Moreover, the radiating elements in the proposed antenna array is metamaterial-inspired for realizing a wider operational bandwidth. The antenna is simply a diskshaped radiator with a rectangular slot and is connected to the ground-plane with a metallic via. The antenna is of subwavelength scale and there is subwavelength separation between the radiating elements. In this configuration the radiating elements behave like resonators or scatters that exhibit metamaterial left-handed properties when excited by electromagnetic fields. The surface impedance matrix elements were obtained as described below.

The boundary conditions here are defined by the current and electric field distributions around the antenna array. In the proposed theoretical modelling, the transverse components of the current distribution have been ignored as we have assumed the size of the radiating patches are less than operating wavelength $(D \ll \lambda)$. Also, the electric field in the groundplane is set to zero.
The surface impedance matrix elements in the $x y$-plane at $z=h$ are specified by the surface impedances matrix of zone I $(z>h)$ (dielectric space) $\left[Z^{(1)}\right]$ and the matrix of zone II $(z<h)$ (MTM substrate layer) $\left[Z^{(2)}\right]$, and is defined by:

$$
Z_{m n}^{(12)}=Z_{m n}^{(1)}-Z_{m n}^{(2)} \quad(m, n=1,2)
$$

The surface impedance matrix is:

$$
\left[\begin{array}{l}
G_{a}^{(1,2)} \\
G_{b}^{(1,2)}
\end{array}\right]=\left[\begin{array}{ll}
Z_{11}^{(1,2)} & Z_{12}^{(1,2)} \\
Z_{21}^{(1,2)} & Z_{22}^{(1,2)}
\end{array}\right]\left[\begin{array}{l}
Q_{a}^{(1,2)} \\
Q_{b}^{(1,2)}
\end{array}\right]
$$

where $G_{a}^{(1,2)}, G_{b}^{(1,2)}$ and $Q_{a}^{(1,2)}, Q_{b}^{(1,2)}$ are the Fourier transforms of the tangential components of the electric and magnetic-fields, respectively, on the radiating elements components in the $x y$-plane at $z=h$ of zone II $(z<h)$ and $G_{a}^{(2)}, G_{b}^{(2)}, Q_{a}^{(2)}$ and $Q_{b}^{(2)}$ are the Fourier transforms of the electric and magnetic-fields tangential components in the plane $z=h$ of zone II $(z>h)$.

It is necessary to first define the surface impedance elements of the metamaterial layer in zone I $(z<h)$. The formulations for the complex amplitudes are given by:

$$
\begin{aligned}
& \frac{\partial H_{y}^{(2)}}{\partial z}-\frac{\partial H_{z}^{(2)}}{\partial y}= \pm k \delta H_{x}^{(2)}+i \omega \varepsilon_{0} \varepsilon_{2} E_{x}^{(2)} \\
& \frac{\partial E_{y}^{(2)}}{\partial z}-\frac{\partial E_{z}^{(2)}}{\partial y}= \pm k \delta E_{x}^{(2)}-i \omega \mu_{0} \mu_{2} H_{x}^{(2)} \\
& \frac{\partial H_{z}^{(2)}}{\partial x}-\frac{\partial H_{x}^{(2)}}{\partial z}= \pm k \delta H_{y}^{(2)}+i \omega \varepsilon_{0} \varepsilon_{2} E_{y}^{(2)} \\
& \frac{\partial E_{z}^{(2)}}{\partial x}-\frac{\partial E_{x}^{(2)}}{\partial z}= \pm k \delta E_{y}^{(2)}-i \omega \mu_{0} \mu_{2} H_{y}^{(2)} \\
& \frac{\partial H_{x}^{(2)}}{\partial y}-\frac{\partial H_{y}^{(2)}}{\partial x}= \pm k \delta H_{z}^{(2)}+i \omega \varepsilon_{0} \varepsilon_{2} E_{z}^{(2)} \\
& \frac{\partial E_{x}^{(2)}}{\partial y}-\frac{\partial E_{y}^{(2)}}{\partial x}= \pm k \delta E_{z}^{(2)}-i \omega \mu_{0} \mu_{2} H_{z}^{(2)}
\end{aligned}
$$

where $k=\sqrt{\varepsilon_{0} \mu_{0}} \omega$ is the wave number for a plane wave in vacuum, with angular frequency defined by $\omega$. Expressions (3)-(8) are specific to the metamaterial domain in Fig.1 that comprises dielectric substrate with an arrangement of slotted circular patches implemented on the substrate surface. The patches are grounded through metallic via to the backside of the substrate layer. The EM field in the metamaterial substrate are defined by these two equations:

$$
\begin{gathered}
\mp m \omega \varepsilon_{0} \varepsilon_{2} \delta \vec{E}+\delta k^{2}\left(-\delta^{2}+\mu_{2} \varepsilon_{2}\right) \vec{H}-\nabla^{2} \vec{H}=0 \\
\pm m \omega \mu_{0} \mu_{2} \delta \vec{H}+\delta k^{2}\left(-\delta^{2}+\mu_{2} \varepsilon_{2}\right) \vec{E}-\nabla^{2} \vec{E}=0
\end{gathered}
$$

It can be shown that by applying the electric and magnetic field derived in [12] into (9) and (10), we can determine the matrix elements of the surface impedance at the plane $z=h$ resulting from zone II $(z<h)$ (MTM substrate layer). Elements of the matrix of surface impedance are given by:

$$
\begin{aligned}
Z_{11}^{(2)}(\alpha, \xi) & =\frac{\cos \left(\zeta_{L} z\right)-\cos \left(\zeta_{R} z\right)}{\left[Z_{12}^{(2)}(\alpha, \xi) \partial^{-}(\alpha, \xi)+m \omega \mu_{0} \mu_{2} \varrho^{-}(\alpha, \xi)\right] l(\xi)} \\
Z_{12}^{(2)}(\alpha, \xi) & =\frac{\cos \left(\zeta_{L} z\right)-\cos \left(\zeta_{R} z\right)}{n l(\xi) Z_{22}^{(2)}(\alpha, \xi)} \partial^{-}(\alpha, \xi)
\end{aligned}
$$




$$
\begin{aligned}
& Z_{21}^{(2)}(\alpha, \xi)=-\frac{m \omega \varepsilon_{0} \varepsilon_{2} \varrho^{+}(\alpha, \xi)}{\partial^{+}(\alpha, \xi)} \\
& Z_{22}^{(2)}(\alpha, \xi)=-\frac{n \varrho^{-}(\alpha, \xi) l(\xi) \omega \varepsilon_{0} \varepsilon_{2}}{\cos \left(\varsigma_{L} z\right)+\cos \left(\varsigma_{R^{2}}\right)}
\end{aligned}
$$

where

$$
\begin{aligned}
& l(\xi)=\left[\left(\frac{-2 \mu_{2} \varepsilon_{2} k^{2} \delta^{2}}{\left(-k^{2} \xi^{2}+\xi^{2}+\mu_{2} \varepsilon_{2} k^{2}\right)^{2}}+1\right)\left(\frac{1}{\left(k^{2} \mu_{2} \varepsilon_{2}-k^{2} \delta^{2}+\xi^{2}\right)}\right)\right]^{-1} \\
& \varrho^{+}(\alpha, \xi)=\left( \pm\left(\zeta_{L} \sin \left(\zeta_{L} Z\right)-\right.\right. \\
& \left.\left.\zeta_{R} \sin \left(\zeta_{R} Z\right)\right)\right)\left(-\frac{\delta^{2} k^{2}}{\left(\delta^{2} k^{2}+\xi^{2}+\mu_{2} \varepsilon_{2} k^{2}\right)}+1\right)-\left(\cos \left(\zeta_{L} z\right)+\right. \\
& \left.\cos \left(\zeta_{R} z\right)\right)\left(-\frac{2 \delta k}{\left(\delta^{2} k^{2}+\xi^{2}+\mu_{2} \varepsilon_{2} k^{2}\right)}\right) \alpha \xi \\
& \varrho^{-}(\alpha, \xi)=-\left(\zeta_{L} \sin \left(\zeta_{L} Z\right)+\right. \\
& \left.\zeta_{R} \sin \left(\zeta_{R} z\right)\right)\left(-\frac{\delta^{2} k^{2}}{\left(\delta^{2} k^{2}+\xi^{2}+\varepsilon_{2} \mu_{2} k^{2}\right)}+1\right) \pm\left(\cos \left(\zeta_{L} z\right)-\right. \\
& \left.\cos \left(\zeta_{R} z\right)\right)\left(-\frac{2 \delta k}{\left(\delta^{2} k^{2}+\xi^{2}+\varepsilon_{2} \mu_{2} k^{2}\right)}\right) \alpha \xi \\
& \partial^{+}(\alpha, \xi)=\left( \pm\left(\cos \left(\zeta_{L} z\right)-\cos \left(\zeta_{R} z\right)\right)\right) \alpha \xi \pm\left(\left(\zeta_{L} \sin \left(\zeta_{L} z\right)+\right.\right. \\
& \left.\left.\zeta_{R} \sin \left(\zeta_{R} z\right)\right)\right)\left(\frac{2 k^{2} \varepsilon_{2} \mu_{2} \delta}{\left(\delta^{2} k^{2}+\xi^{2}+\varepsilon_{2} \mu_{2} k^{2}\right)}+k \delta\right) \\
& \partial^{-}(\alpha, \xi)=\left(\cos \left(\zeta_{L} z\right)+\cos \left(\zeta_{R} z\right)\right) \alpha \xi \pm\left(\zeta_{L} \sin \left(\zeta_{L} z\right)-\right. \\
& \left.\zeta_{R} \sin \left(\zeta_{R} z\right)\right)\left(\frac{2 k^{2} \varepsilon_{2} \mu_{2} \delta}{\left(\delta^{2} k^{2}+\xi^{2}+\varepsilon_{2} \mu_{2} k^{2}\right)}+k \delta\right)
\end{aligned}
$$

In a similar way we can determine the expressions for the elements of the matrix of surface impedances at $z=h$ resulting from zone I $(z>h)$ (dielectric space) by applying the electric and magnetic field expressions derived in [12] into (9) and (10). Elements of the matrix of surface impedance are given by:

$$
\begin{aligned}
& Z_{11}^{(1)}=\left(\sqrt{\alpha^{2}+\xi^{2}-\mu_{1} \varepsilon_{1} k^{2}}\right)\left(\frac{\mu_{0} \mu_{1} \omega}{\xi^{2}+\mu_{1} \varepsilon_{1} k^{2}}\right) \\
& Z_{12}^{(1)}=\left(\sqrt{\alpha^{2}+\xi^{2}-\mu_{1} \varepsilon_{1} k^{2}}\right)\left(\frac{\mu_{0} \mu_{1} \omega}{\alpha \xi}\right) \\
& Z_{21}^{(1)}=-\left(\sqrt{\alpha^{2}+\xi^{2}-\mu_{1} \varepsilon_{1} k^{2}}\right)\left(\frac{\mu_{0} \mu_{1} \omega}{\xi \alpha}\right) \\
& Z_{22}^{(1)}=-\left(\sqrt{\alpha^{2}+\xi^{2}-\mu_{1} \varepsilon_{1} k^{2}}\right)\left(\frac{\mu_{0} \mu_{1} \omega}{\alpha^{2}+\mu_{1} \varepsilon_{1} k^{2}}\right)
\end{aligned}
$$

Elements of the matrix for surface impedances in the $x y$ plane $z=h$ can also be determined in a similar way as described above. Then by performing inverse Fourier transform on equation (1) and applying appropriate boundary conditions we can obtain formulations relating the electric and magnetic fields with a current distribution at the substrate surface $z=h$. The above formulations are valid for the metamaterial domain and they have no restriction on the dimensions, conductor or dielectric layer thickness, values of permittivity or permeability.

The above theoretical model applies specifically to the single layer metamaterial-inspired antenna array structure shown in Fig.1. The model was validated by comparing it with CST Studio Suite ${ }^{\circledR}$ which is a commercial 3D full-wave EM analysis software. For the purpose of the validation the dimensions of the array are thus: dielectric substrate $=$ $40 \times 40 \times 5 \mathrm{~mm}^{3}$, radius of circular patches $=3 \mathrm{~mm}$, length of slot $=4 \mathrm{~mm}$, width of the slot $=0.5 \mathrm{~mm}$, radius of via-holes $=$ $0.4 \mathrm{~mm}$, inter-space gap $(s)=4 \mathrm{~mm}$, and gap between arrays $(g)=10 \mathrm{~mm}$. When the antenna array is exposed to EM fields surface currents are induced. The surface current distribution over the antenna array at various frequencies can be visualized using the EM software, as shown in Fig.2. It shows the current is distributed over the patch antennas and a close examination reveals that the current is more strongly distributed around the slots and over the metallic vias that connect the disk to the ground-plane.

The surface impedances in zones I and II predicted by the theoretical model and EM software are given in Figs. 3 and 4 over frequency range from 3-5 GHz. It is evident from these results there is total agreement between the proposed theoretical model and EM software. This shows effectiveness of the proposed formulations. The theoretical model was benchmarked against EM software by CST Studio Suite ${ }^{\circledR}$ using the same central processing unit. Computational time of theoretical model was 125 seconds and using the EM software was 205 seconds. The theoretical model is significantly less computationally intensive by $61 \%$.

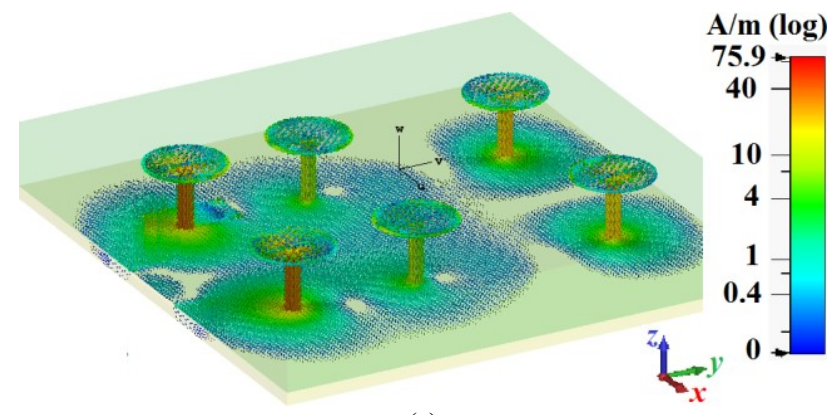

(a)

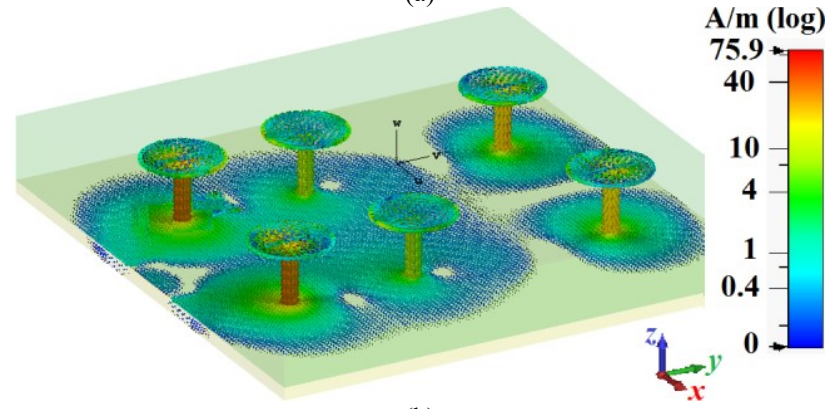

(b)

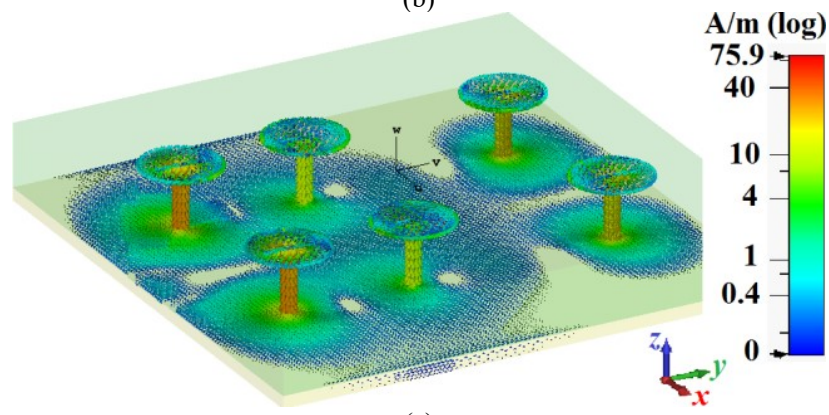

(c)

Fig.2. The current distribution in the patch array at various spot frequencies, (a) $3 \mathrm{GHz}$, (b) $4 \mathrm{GHz}$, and (c) $5 \mathrm{GHz}$.

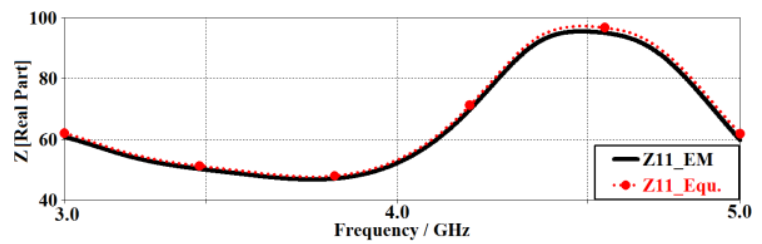


(a) Real part of $Z_{11}$

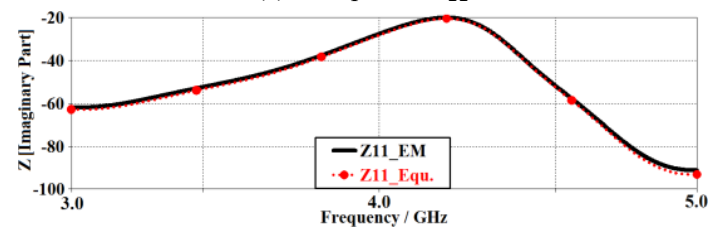

(b) Imaginary part of $Z_{11}$

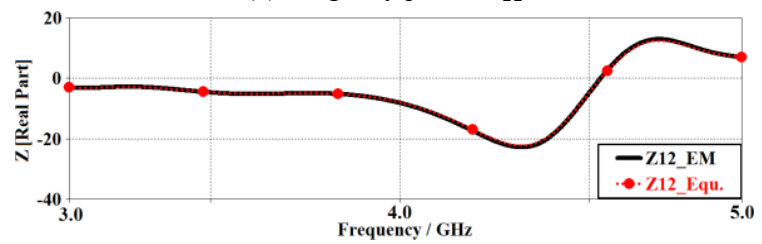

(c) Real part of $Z_{12}$

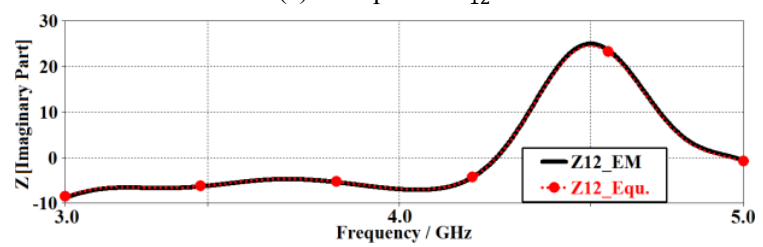

(d) Imaginary part of $Z_{12}$

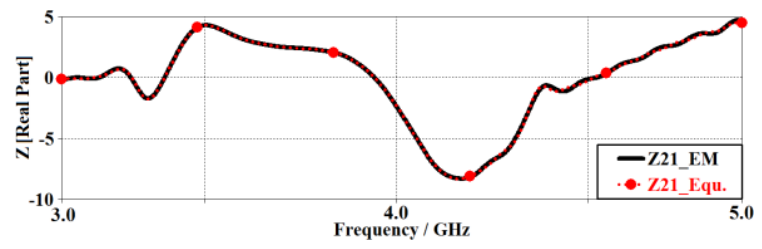

(e) Real part of $Z_{21}$

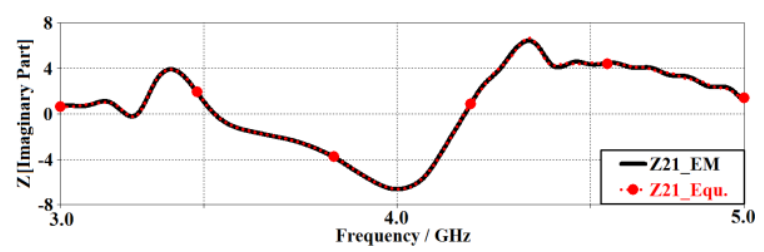

(f) Imaginary part of $Z_{21}$

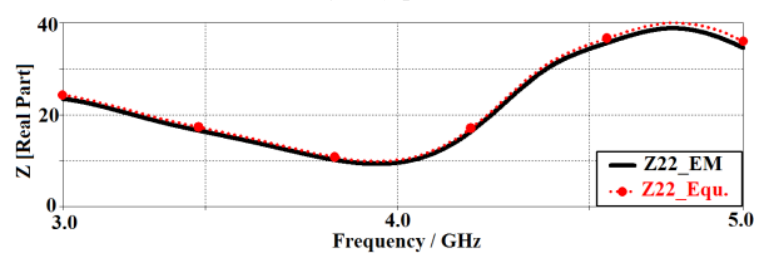

(g) Real part of $Z_{22}$

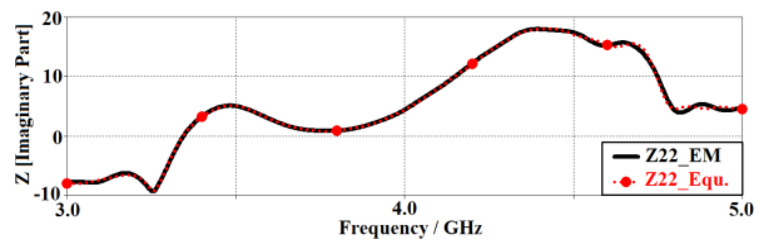

(h) Imaginary part of $Z_{22}$

Fig.2. Surface impedance at the plane $z=h$ of zone I $(z>h)$ (dielectric space) using the EM software and proposed theoretical formulations.

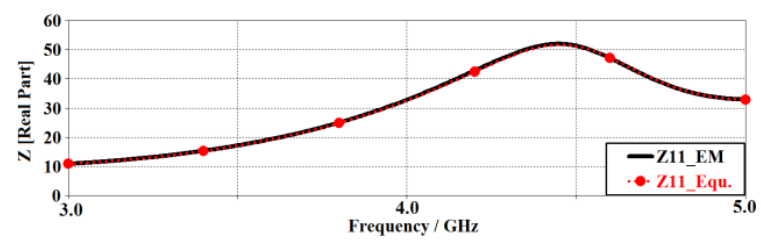

(a) Real part of $Z_{11}$

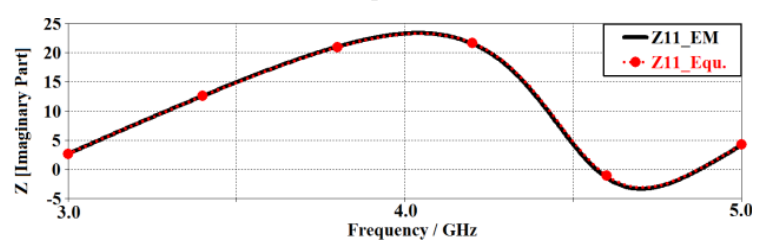

(b) Imaginary part of $Z_{11}$

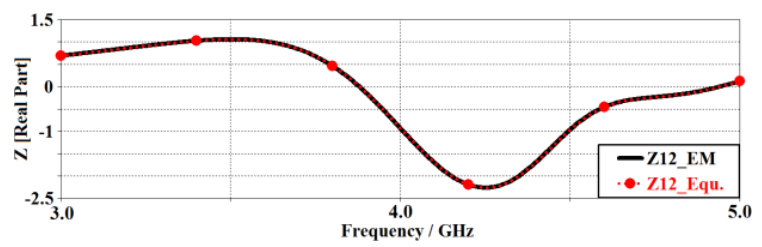

(c) Real part of $Z_{12}$

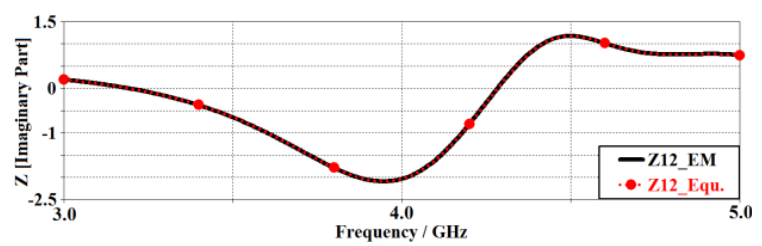

(d) Imaginary part of $Z_{12}$

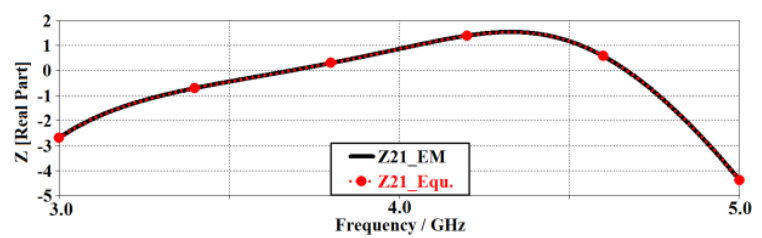

(e) Real part of $Z_{21}$

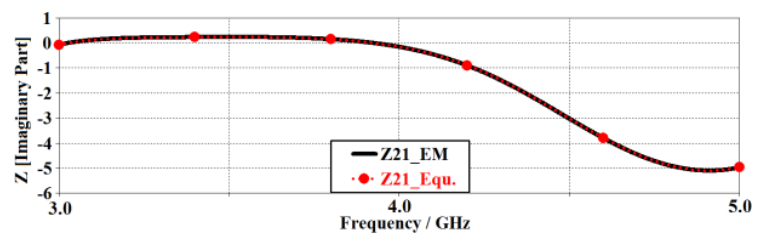

(f) Imaginary part of $Z_{21}$

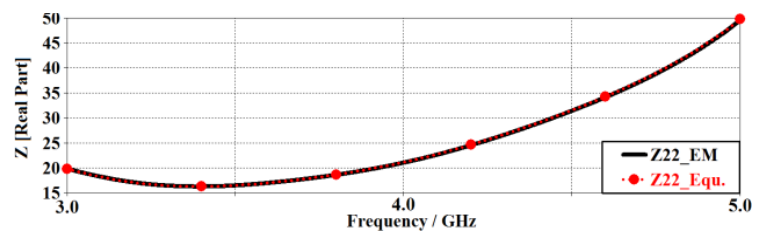

(g) Real part of $Z_{22}$

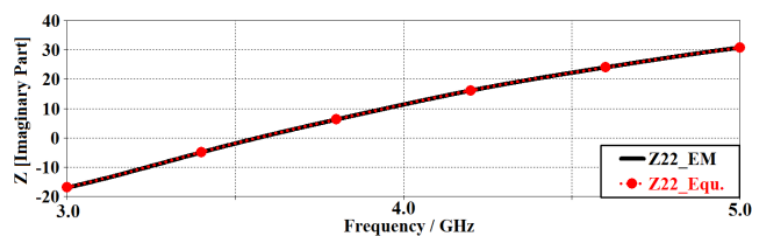

(h) Imaginary part of $Z_{22}$

Fig.3. Surface impedances at the plane $z=h$ of zone II $(z<h)$ (MTM layer) using the EM software and proposed theoretical formulations.

\section{CONCLUSION}

A highly accurate theoretical model is presented for electrodynamic analyses of an antenna array implemented on a dielectric substrate. The theoretical model is applied to an array consisting of an arrangement of slotted circular patch radiators each 
of which are grounded with metallic vias. The theoretical model was benchmarked against a commercial 3D full wave electromagnetic solver based on MoM and using the same central processing unit. The proposed methodology is shown to be accurate and uses significantly less computational time. There is excellent agreement of the surface impedance results from both methodologies over a specified frequency range. The accuracy of the theoretical model presented is valid for single layer structures. The model needs to be extended for multilayer structures.

\section{REFERENCES}

[1] W. Zhang, Y. Liu, Y. Ji, "Circularly polarized antenna array with low RCS using metasurface-inspired antenna units," IEEE Antennas and Wireless Propagation Letters, Vol. 18, No. 7, July 2019, pp. 1453-1457.

[2] M. Mohammadi, et al., "Backfire-to-endfire scanning capability of a balanced metamaterial structure based on slotted ferrite-filled waveguide", Waves in Random and Complex Media, https://doi.org/10.1080/17455030.2019.1654148, pp.1-15, 2019.

[3] M. Mohammadi, et al., "A partially ferrite-filled rectangular waveguide with CRLH response and its application to a magnetically scannable antenna," Journal of Magnetism and Magnetic Materials, Vol.491, 1 Dec. 2019, 165551, https://doi.org/10.1016/j.jmmm.2019.165551.

[4] M. Alibakhshikenari, et al., "Extended aperture miniature antenna based on CRLH metamaterials for wireless communication systems operating over UHF to C-band," Radio Science, Vol. 53, Issue 2, Feb. 2018, pp.154-165.

[5] M. M. Shirkolaei, J. Ghalibafan, "Unbalanced CRLH Behavior of Ferrite-Loaded Waveguide Operated Below Cutoff Frequency," Waves in Random and Complex Media, 2020, pp. 1-16, Doi:10.1080/17455030.2020.1800133.

[6] A. Jafargholi, et al., "Mutual coupling reduction in an array of patch antennas using CLL metamaterial superstrate for MIMO applications," IEEE Trans. on Ants and Pro, Vol. 67, No. 1, Jan. 2019, pp. 179-189.

[7] A. Iqbal, O. A. Saraereh, A. Bouazizi, and A. Basir, "Metamaterialbased highly isolated MIMO antenna for portable wireless applications," Electronics, 7, No. 10 (2018): 267.

[8] M. Alibakhshikenari, et al., "Isolation enhancement of densely packed array antennas with periodic MTM-photonic bandgap for SAR and MIMO systems," IET Microwaves, Antennas \& Propagation, Vol.14, Issue 3, February 2020, pp. 183 - 188.

[9] B. Feng, et al., "A dual-wideband and high gain magneto-electric dipole antenna and its 3D MIMO system with metasurface for 5G/WiMAX/WLAN/X-band applications," IEEE Access, Vol. 6, 2018, pp. 33387-33398.

[10] M. Alibakhshikenari, et. al., "Surface wave reduction in antenna arrays using metasurface inclusion for MIMO and SAR systems," Radio Science, 54, 2019, 1067-1075.

[11] I. V. Lindell, "Variational method for the analysis of lossless Biisotropic (nonreciprocal chiral) waveguides," IEEE Trans. Microwave Theory Tech., Vol. 40, Issue 2, 1992, pp. 402-405.

[12] D. Klyuev, et al., "Mathematical modeling of multi-element antenna arrays with chiral metamaterials substrates using singular integral equations," EPJ Web Conf., IV International Conference, section on Modeling of Nonlinear Processes and Systems, Vol. 224(1), article number 02002, 8 pages, December 2019.

[13] C. Caloz and T. Itoh, Electromagnetic Metamaterials: Transmission Line Theory and Microwave Applications, Wiley-IEEE Press, 7 November 2005, 376 Pages. 